\title{
Instrumented ball design and development
}

Suda Martins, Wei Li, Peter Radziszewski

Department of Mechanical Engineering

McGill University

In the mineral processing industry, tumbling mills are used in the size reduction or comminution of ore to a target size distribution. As the tumbling mill environment is quite violent it is difficult to monitor mill charge motion let alone single ball or particle motion. An effort was initiated to investigate the development of an instrumented ball that includes data collection capabilities. A variety of sensors, including accelerometers and gyros, form the instrument package. The data collected by the instruments allow the calculation of a number of dynamic and kinematic quantities as a function of time. Using instrumented ball data, the error model of the instrument can be found. Finally, the importance of the data with respect to ball mills is discussed along with the relationship between the instrumented ball data and charge motion simulation results is discussed. 